\title{
Correction to: A historical geomorphological approach to flood hazard management along the shore of an alpine lake (northern Italy)
}

\author{
F. Luino ${ }^{1}$ (D) A. Belloni ${ }^{2} \cdot$ L. Turconi $^{1} \cdot$ F. Faccini $^{3} \cdot$ A. Mantovani ${ }^{1}$ • \\ P. Fassi ${ }^{4} \cdot$ F. Marincioni ${ }^{5} \cdot$ G. Caldiroli ${ }^{2}$ \\ Published online: 24 July 2018 \\ (C) Springer Nature B.V. 2018
}

\section{Correction to: Natural Hazards https://doi.org/10.1007/s11069-018-3398-5}

This correction stands to correct mistakes presented in the original article due to a lag in the e-proofing system and the correction handling for this article. The original article has been corrected.

The original article can be found online at https://doi.org/10.1007/s11069-018-3398-5.

F. Luino

fabio.luino@irpi.cnr.it

1 Consiglio Nazionale delle Ricerche, Istituto di Ricerca per la Protezione Idrogeologica (CNRIRPI), Strada delle Cacce 73, 10135 Turin, Italy

2 Regione Lombardia, Direzione Generale, Sicurezza, Protezione Civile e Immigrazione, Piazza Lombardia 1, 20100 Milan, Italy

3 DISTAV - Universita' di Genova, Corso Europa 26, 16145 Genoa, Italy

4 Regione Lombardia, Protezione Civile, Coordinamento Service Tecnico H24, Sala Operativa, 20100 Milan, Italy

5 Dipartmento di Scienze della Vita e dell'Ambiente, Universita' Politecnica delle Marche, Via Brecce Bianche 12, SNC, 60131 Ancona, Italy 